\title{
Microstudy of the Anisotropy of Sandy Material
}

\author{
Kunyong Zhang $\mathbb{D}^{1,2}$ Zhenjun Zang $\mathbb{D}^{1,2}$ Leslie Okine, ${ }^{1,2}$ and Jose Luis Chavez Torres $\mathbb{D}^{1,2}$ \\ ${ }^{1}$ Key Laboratory of Ministry of Education for Geomechanics and Embankment Engineering, Hohai University, \\ Nanjing 210098, China \\ ${ }^{2}$ Institute of Geotechnical Engineering, Hohai University, Nanjing 210098, China
}

Correspondence should be addressed to Kunyong Zhang; ky_zhang@hhu.edu.cn

Received 21 May 2018; Accepted 9 July 2018; Published 14 August 2018

Academic Editor: Xinbao Yu

Copyright (c) 2018 Kunyong Zhang et al. This is an open access article distributed under the Creative Commons Attribution License, which permits unrestricted use, distribution, and reproduction in any medium, provided the original work is properly cited.

\begin{abstract}
Through numerical simulation based on the particle flow method, DEM numerical test samples were generated with the results of laboratory tests on standard sand. The method consists of the use of gravity deposition and radius expansion modeling of irregular sand particles, where samples of the biaxial test are assembled by generated long particle units. Different steps of deposition or initial stresses were applied during the sample generation process in order to simulate different sample states. The loads from the horizontal and vertical directions are, respectively, applied to samples, and then the stress-strain curve and macroscopic mechanical parameters are acquired. The numerical experiment results show that the gravitational deposits have significant impact on the major axis orientation arrangement of particles and on the average coordination number, as well as the initial stress has a significant effect on it. There is a remarkable effect on the stress-strain curve and on the acquired mechanical parameters as a result of the application of load to samples from the horizontal and vertical directions. The sand samples show an obvious property of inherent anisotropy and stress-induced anisotropy.
\end{abstract}

\section{Introduction}

Sand is composed of discrete particles. The gradation, shape, size, and physical properties of particles determine the macromechanical properties of sand under different stress conditions. Anisotropy is a typical characteristic of sand.

There are two types of sand anisotropy: inherent anisotropy and stress-induced anisotropy. Inherent anisotropy means that irregular particles show a tendency of directional arrangement because its particle structure is influenced by external factors like gravity and water in the deposition process. This directional arrangement has an effect on the microstructure of the sand and also shows the anisotropy in the macroscopic mechanical properties [1-3]. Stressinduced anisotropy means that the change of the stress state leads to the change of the mechanical properties and parameters of the sand when complex load is applied in different directions $[4,5]$. Considering the microscopic mechanism of anisotropy, both the inherent anisotropy and stress-induced anisotropy can be attributed to the structural properties of the material itself. As sand particles have a nonspherical structure, this makes the particle arrangement, contact, and microstructure change under the action of the external load, which results in a macroscopic anisotropy.

From the macroscopic view, the main reason for the inherent anisotropy of sand is that the particles are arranged along the principal stress direction during the gravitational deposition process, and finally, the difference between the vertical and horizontal mechanical properties is formed on the macroscopic scale. The inherent anisotropy can be described by the elastic theory: vertical elastic modulus $E_{\mathrm{v}}$ and Poisson's ratio $v_{\mathrm{v}}$, horizontal elastic modulus $E_{\mathrm{h}}$ and Poisson's ratio $v_{\mathrm{h}}$, and shear strength $G$. In addition to the above, the vertical modulus $E_{\mathrm{v}}$ is greater than the horizontal modulus $E_{\mathrm{h}}$. In terms of stress-induced anisotropy, Zhang and Yin [6] found that the stress-strain relationship of the soil under the complex stress state shows significant stress anisotropy, based on the true triaxial test. The internal mechanism of the inherent anisotropy and the stress-induced anisotropy is that 
the external conditions (gravity, load, water, etc.) change the microstructure of the soil, and thus, the soil shows different stress and deformation characteristics macroscopically. Based on the true triaxial test, Hoque and Tatsuoka [7] also concluded that the elastic modulus in the horizontal and vertical directions of the soil showed significant anisotropy under nonisotropic loading.

PFC2D (particle flow code in 2 dimensions) [8] is the general-purpose code based on discrete element method (DEM) put forward by Cundall [9], which simulates movement and interaction of granular media, confirming particle's location and velocity in every moment according to the motion equation of translator motion and rotation. In this paper, PFC2D was used to establish several sets of irregular sand particles, to reveal the formation and development mechanism of soil anisotropy, by simulating the biaxial compression numerical test of standard sand, and loading from the horizontal and vertical directions, respectively. Then, the stress-strain curve and the mechanical parameters were obtained in different directions, and the microscopic mechanism of two anisotropic conditions is explained by the micromechanic parameters such as the average coordination number and the normal contact force of the particles.

\section{Introduction of the PFC2D and the Numerical Specimen}

2.1. DEM and PFC2D. Most of the former laboratory experiments and theoretical studies on soil anisotropy have assumed that the soil is a continuous and isotropic medium. Limited to the theoretical basis and experimental conditions, microanalysis of the structural causes of soil anisotropy cannot be carried out. DEM and PFC provide the conditions for microanalysis on the basis of traditional continuous medium research $[10,11]$.

The macroscopic mechanical behavior of the material analyzed in PFC was conducted by studying the microscopic parameters of the particles and obtaining the relationship between the microstructure and the macroscopic mechanical behavior. Chi [12] used PFC to simulate the formation and development of sand shear zone, and the principle governing sand was obtained under different confining stress. Jiang et al. [13] studied the formation mechanism of the soil shear zone by discrete element simulation of the plane strain biaxial test. Tong et al. [14] used the DEM to analyze the microstructures of anisotropic particles from the orientation of the major axis and void ratio of the particles. These studies have analyzed the effect of the change of microstructure on the macroscopic mechanical behavior of soil, which proved that PFC is an effective and powerful tool. In this paper, PFC2D is also used to study the anisotropic behaviors of sands under complicated stress states.

\subsection{Method for Numerical Sample Preparation}

\subsubsection{The Calibration of Numerical Sample Parameters}

(1) The Results of Fujian Standard Sand in Laboratory Tests. This paper is based on the laboratory triaxial test of Fujian sand [15] to calibrate the parameters. Fujian standard sand is a kind of well-graded medium sand. The macroscopic parameters obtained in triaxial tests are shown in Table 1.

In Table $1, E_{50}$ is the secant deformation modulus, corresponding to the slope of the stress-strain curve at half the peak intensity. $v_{50}$ is the secant Poisson's ratio, which is on the lateral strain-loading direction strain curve, corresponding to half of the peak intensity cut line ratio, and $\varphi$ is the internal friction angle calculated from the peak intensity of the stress-strain curve. They are calculated using following equations:

$$
\begin{aligned}
E_{50} & =\left(\frac{\Delta \sigma_{1}}{\Delta \varepsilon_{1}}\right)_{50}, \\
v_{50} & =\frac{1}{2}\left[1-\left(\frac{\Delta \varepsilon_{\mathrm{v}}}{\Delta \varepsilon_{1}}\right)_{50}\right], \\
\varphi & =\arcsin \left(\frac{\sigma_{1 \mathrm{f}}-\sigma_{3 \mathrm{f}}}{\sigma_{1 \mathrm{f}}+\sigma_{3 \mathrm{f}}}\right),
\end{aligned}
$$

where $\Delta \sigma_{1}$ is the major principal stress increment, $\Delta \varepsilon_{1}$ is the strain increment in the direction of the major principal stress, $\Delta \varepsilon_{\mathrm{v}}$ is the volumetric strain increment, and $\sigma_{1 \mathrm{f}}$ and $\sigma_{3 \mathrm{f}}$ are the major principal stress and the minor principal stress at the peak intensity, respectively.

(2) Numerical Test Environment. The calibration of the microscopic parameters is determined by comparing the numerical results with the laboratory test results. The results of the laboratory test in this paper are the results of the triaxial test of Fujian standard sand described above. The numerical sample is a two-dimensional specimen, and PFC2D was used to simulate the biaxial test. The model for the numerical sample is shown in Figure 1.

$h$ and $w$ represent the height and width of the numerical sample, respectively.

(3) The Generation and Loading of Numerical Samples. Original PFC2D particles are standard discs, which differ greatly from the irregular shape of the actual sand particles. In order to simulate the irregular structure of the actual sand particles, several disc particles were assembled into a complex shape block, and relative deformation and displacement between the disc particles were limited. Only the contact between the blocks was taken into account without regarding the contact and force transfer between the disc particles constituting the block. The soil particles used in this paper are composed of three discs, and the comparison with the standard sand is shown in Figure 2. The center of the three disc particles is on the same line, and the angle $\theta$ is the major axis orientation angle of the irregular element (the angle between the horizontal axis and that through the center line of the irregular element).

The numerical sample is formed by the radius expansion method as described by the following steps:

(1) Two-dimensional space was generated having a width and height of $50 \mathrm{~mm}$ and $105 \mathrm{~mm}$, 
TABLE 1: Macroscopic parameters of Fujian sand in laboratory tests.

\begin{tabular}{llc}
\hline$E_{50}(\mathrm{MPa})$ & $v_{50}$ & $\varphi\left(^{\circ}\right)$ \\
\hline 44.3 & 0.3 & 41.2 \\
\hline
\end{tabular}

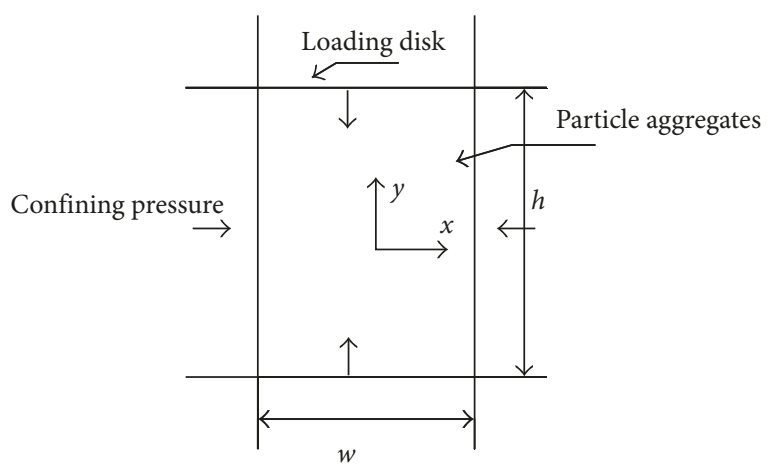

FIgure 1: Model for the numerical sample.

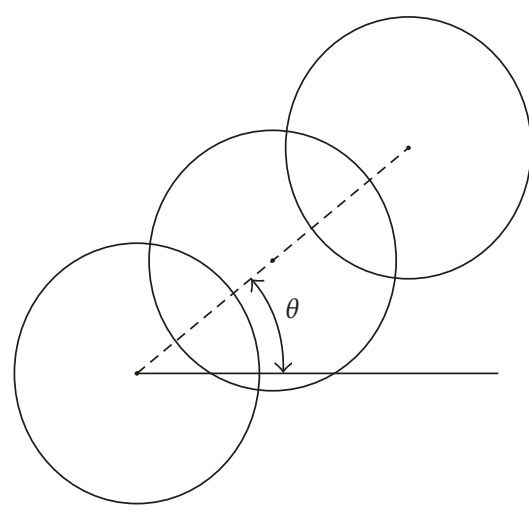

(a)

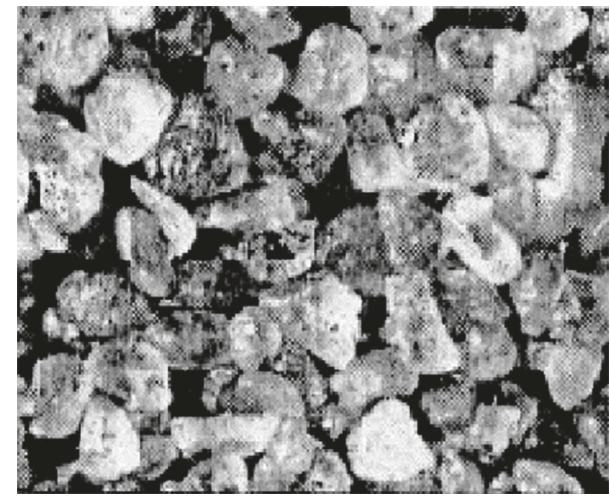

(b)

Figure 2: Comparison between irregular element and Fujian sand.

respectively, as shown in Figure 3(a), and the radius expansion method was used to generate disc particles with parameters, as shown in Table 2. The disc particles were evenly distributed, as shown in Figure 3(b). In order to reduce the effect of particle size, the ratio of the size of the biaxial specimen to the particle size satisfies $L / R>30$, so the minimum and maximum particle sizes of the particles were kept as $0.6 \mathrm{~mm}$ and

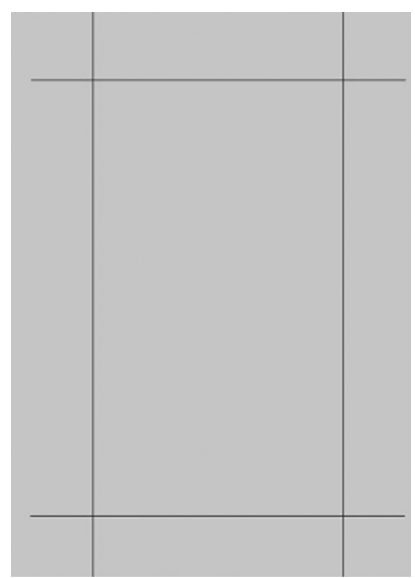

(a)

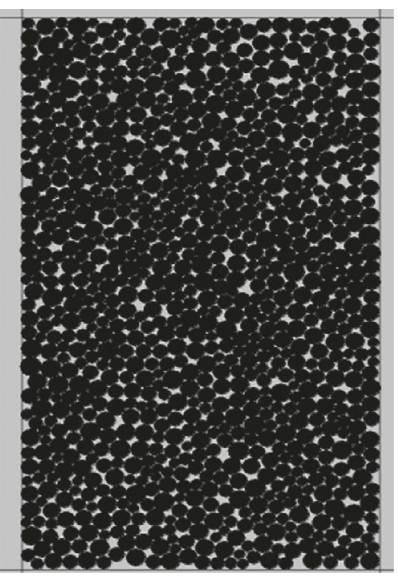

(b)

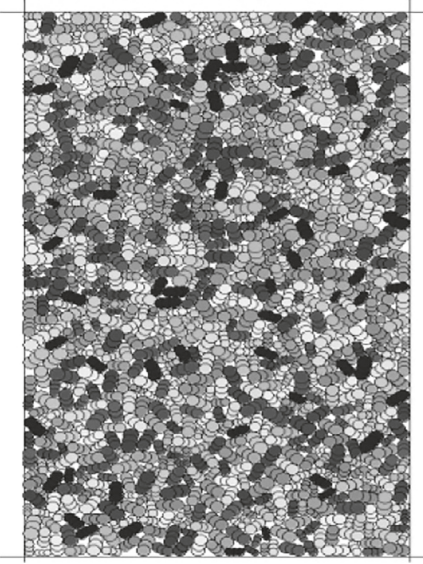

(c)

FIgURE 3: Process of generating numerical samples: (a) generating wall; (b) generating disc unit samples; (c) generating long unit samples.

$1.2 \mathrm{~mm}$. The porosity area was 0.15 , and its macroscopic mechanical properties were close to the dense sand.

(2) A program was coded using the FISH language in PFC to record the center position and radius of the two-dimensional disk particles, and then the disc 
TABLE 2: Microscopic parameters of samples at the initial state.

\begin{tabular}{lccccc}
\hline$k_{\mathrm{n}}(\mathrm{Pa})$ & $k_{\mathrm{n}} / k_{\mathrm{s}}$ & $f$ & $R_{\max }(\mathrm{mm})$ & $R_{\min }(\mathrm{mm})$ & $n$ \\
\hline $2 e 8$ & 2 & 0.5 & 1.2 & 0.6 & 0.18
\end{tabular}

Note: $k_{\mathrm{n}}$ is the normal stiffness of the particles, $k_{\mathrm{s}}$ is the tangent stiffness of the particles, $k_{\mathrm{n}} / k_{\mathrm{s}}$ is the tangential stiffness ratio, $f$ is the coefficient of friction, $R_{\max }$ is the maximum radius of the particles, $R_{\min }$ is the minimum radius of the particles, and $n$ is the porosity (area porosity).

particles were removed. In the same position, the irregular elements were generated according to the principle of "Area and mass equivalent." The major axis orientation of the irregular elements was $0^{\circ}$ to $180^{\circ}$ randomly distributed, as shown in Figure 3(c).

After the test was completed, the confining pressure and the load were applied and subjected to a biaxial test. By adjusting the initial sample parameters in Table 2, the macroscopic mechanical properties exhibited by the sample were consistent with the laboratory triaxial test results.

2.2.2. Parameter Calibration Results. The initial microscopic parameters were adjusted until the macroscopic parameters of the model were similar to the macroscopic parameters obtained by the ordinary triaxial test. The final microscopic parameters of the standard sand in Fujian are shown in Table 3.

The macroscopic mechanical parameters, axial stress, and axial strain curves obtained from numerical experiments and the comparison with laboratory test results are shown in Table 4 and Figure 4.

From Table 4 and Figure 4, the parameter calibration of the irregular unit is basically the same as that of Shi [15] and the laboratory test results. However, from Figure 4, the numerical model results of this paper are closer to the laboratory test results. This also shows that the irregular particle unit is more suitable to simulate the real structure of the sand, and the numerical simulation of Fujian standard sand with the irregular particle unit is more realistic, and therefore, the numerical test of the wider stress path can be carried out on the basis of this sample.

\section{Standard Sand Numerical Test Process and Results}

3.1. Sample Preparation. In order to find out the difference and relationship between the inherent anisotropy and stressinduced anisotropy of the sand, the same method and test environment and parameters were used for the anisotropic numerical samples.

(1) Test Environment and Parameters. The biaxial test is carried out with the experimental environment shown in Figure 2, and the expected size of the specimen was $60 \mathrm{~mm} \times 60 \mathrm{~mm}$. The particle unit of the biaxial specimen was the irregular unit, and the microscopic parameter of the sample particle was calibrated with the parameter, as shown in Table 3.
TABLE 3: Microscopic parameters of samples at the final state.

\begin{tabular}{lccccc}
\hline$k_{\mathrm{n}}(\mathrm{Pa})$ & $k_{\mathrm{n}} / k_{\mathrm{s}}$ & $f$ & $R_{\max }(\mathrm{mm})$ & $R_{\min }(\mathrm{mm})$ & $n$ \\
\hline $1.3 e 8$ & 3 & 0.5 & 1.2 & 0.6 & 0.15 \\
\hline
\end{tabular}

TABle 4: Parameters of numerical simulation and laboratory test.

\begin{tabular}{lccc}
\hline $\begin{array}{l}\text { Fujian standard } \\
\text { sand }\end{array}$ & $\begin{array}{c}\text { Initial modulus } \\
(\mathrm{MPa})\end{array}$ & $\begin{array}{c}\text { Poisson's } \\
\text { ratio }\end{array}$ & $\begin{array}{c}\text { Internal friction } \\
\text { angle }\left(^{\circ}\right)\end{array}$ \\
\hline Laboratory test & 44.3 & 0.30 & 41.2 \\
Shi [15] & 48.4 & 0.34 & 41.0 \\
This article & 43.3 & 0.27 & 40.8 \\
\hline
\end{tabular}

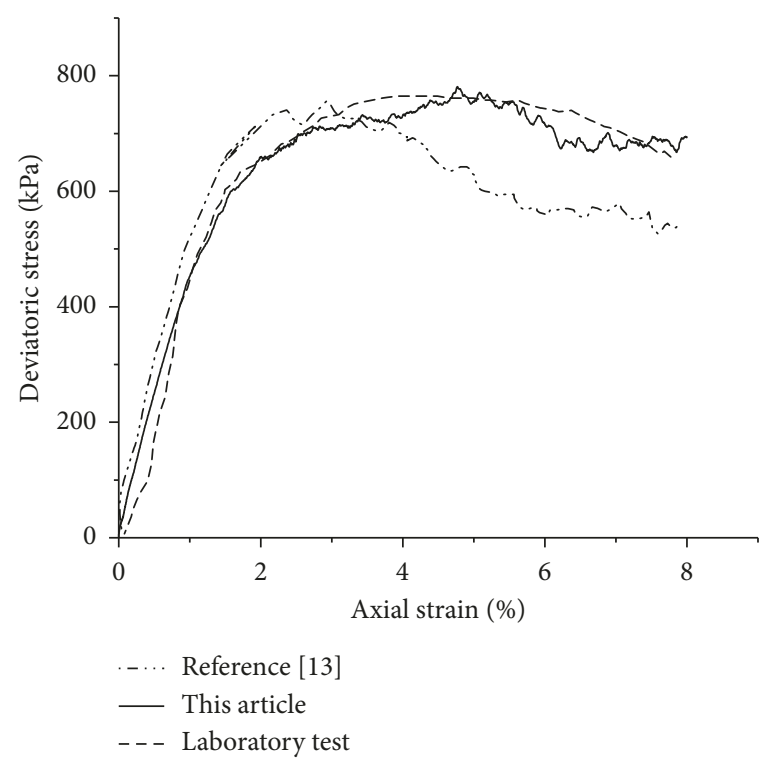

Figure 4: Curves of numerical simulation and laboratory test.

(2) Sample Generation. The two-axis numerical sample generation steps are as follows: firstly, a two-dimensional space of $100 \mathrm{~mm}$ and a width of $60 \mathrm{~mm}$ were generated, and then microscopic parameters were generated in the space. The particle gradation follows a uniformly distributed disc particle, and irregular units were generated by using the program coded in the FISH language in PFC. The initial major axis of the regular element was randomly distributed. The original top wall was deleted, and a new top wall was regenerated at a height of $60 \mathrm{~mm}$. Finally, the particles above the new wall were removed, and the numerical sample was generated.

\subsection{Sample Loading and Control}

3.2.1. The Stress Path of the Inherent Anisotropy. The basic unit used in this test is an irregular unit which is under the action of gravity deposition, and the major axis orientation of particles was arranged in a specific direction. In order to simulate the effect of soil deposition on the microstructure and macroscopic mechanical properties of soil particles, the gravity was applied to the irregular unit, and the sedimentation occurred under the action of gravity. Three sets of 
samples were generated according to the number of gravity deposition sample steps, and then the gravity load was removed. The confining pressure of $100 \mathrm{kPa}$ was applied to make the sample reach the initial equilibrium state. Sample number, the number of deposition steps, and the subsequent loading direction are shown in Table 5.

In Table 5, each set of samples which has the same letter used the same number of gravity deposition steps. A slight initial stress was imposed in the horizontal and vertical directions to keep the sample stable. The stress increment was applied firstly in the horizontal direction and then the vertical direction to compare the macroscopic mechanical properties in the horizontal and vertical directions with the same initial sample. The number of deposition steps in Table 5 refers to the time step in order to make the numerical calculation stable and select the time less than a critical time step, which is related to the frequency of each particle unit.

The numerical test stress path is shown in Figure 5.

\subsubsection{The Stress Path of the Stress-Induced Anisotropy.} In this experiment, six samples were divided into three sets according to the initial stress state (Table 6). The initial stress state of the two samples in each set was the same, and then three groups of samples were loaded from the horizontal and vertical directions, respectively. The stress-strain curves and macroscopic mechanical parameters of the samples in different directions were obtained. The stress path of the numerical test is shown in Figure 6.

3.3. Test Results. The stress-strain curves obtained from the horizontal and vertical loading are shown in Figure 7, and the relative mechanical parameters are shown in Table 7. The initial modulus refers to the stress and strain ratio when the strain of the load direction reaches $1 \%$.

From Figures $7(a)-7(c)$, it can be seen that the stressstrain curves of the three groups show a common principle: the stress peak and the initial modulus at the time of loading in the vertical direction (deposition direction) are larger than those in the horizontal direction. Since the two samples in each set of tests experienced the same number of gravitational steps, they had the same porosity, cell size and sample size, and the initial state. The only difference between the two samples is that the load increment was applied in the horizontal direction for one and the other was loaded from the vertical direction. The macroscopic mechanical response in different directions shows obvious anisotropy. From the stress and strain curves of different deposition steps, it can be noticed that the larger the number of deposition steps, the greater the peak stress.

From Figures 7(d)-7(f), the stress-strain curves obtained from the minimum principal stress direction (horizontal direction) of the three sets are higher than those obtained by loading from the maximum principal stress direction. The initial elastic modulus and the stress peak are larger than those obtained from the maximum principal stress direction. With the increase of the initial stress difference in the horizontal and vertical directions, the difference of the
TABLE 5: List of samples and test parameters.

\begin{tabular}{lcc}
\hline Sample number & Gravity deposition steps & Loading direction \\
\hline A1 & 90000 & Horizontal \\
A2 & & Vertical \\
B1 & 120000 & Horizontal \\
B2 & & Vertical \\
C1 & 150000 & Horizontal \\
C2 & & Vertical \\
\hline
\end{tabular}

relevant mechanical parameters is also more obvious. It shows that the specimen exhibits obvious anisotropy after being subjected to unequal stress.

In the inherent anisotropic soil, the major axis orientation arrangement of the soil particles forms a macroscopic anisotropic layered foundation, making the elastic modulus of the deposition direction (i.e., vertical direction) larger than that of the horizontal direction.

But for the stress-induced anisotropy, the gravitational loading step is not applied, the sample is initially isotropic, and the particle arrangement does not have a major axis oriented arrangement. Because of the initial stress of the primary isotropic specimen, the initial stresses in the direction of maximum principal stress and minimum principal stress are different, resulting in different strain increments when stress increases are applied from different directions, so that the elastic modulus of the maximum principal direction is smaller than that of the direction of minimum principal stress. The difference in the principle of macroscopic stress and deformation is entirely due to the additional stress increment, which is a typical stress-induced anisotropy.

\section{Anisotropic Microscopic Mechanism Analysis}

4.1. Analysis of Microcosmic Mechanism of the Inherent Anisotropy. From the macroscopic mechanical parameters and the stress-strain curves of the numerical experiments, it shows that there is obvious inherent anisotropy in different directions due to gravity deposition. In this paper, by analyzing the change of the major axis orientation and the change of the coordination number of the internal particles in the sample during the loading process, the occurrence and development of the inherent anisotropy were found from the perspective of the microstructure parameters.

4.1.1. The Effect of Major Axis Orientation on the Particles. In order to analyze the specific principles of the microstructures in the gravitational deposition process, a program coded with the FISH language was used to track the changes of the major axis orientation of the particle units before and after gravity deposition. Prior to gravitational deposition, the major axes of the irregularly shaped units are randomly distributed and are approximately equal in each interval. Figures $8(a)-8(c)$ show the major axis distribution of the particles in the three sets of samples. In the figure, the abscissa is the angle between the center line axis of the 


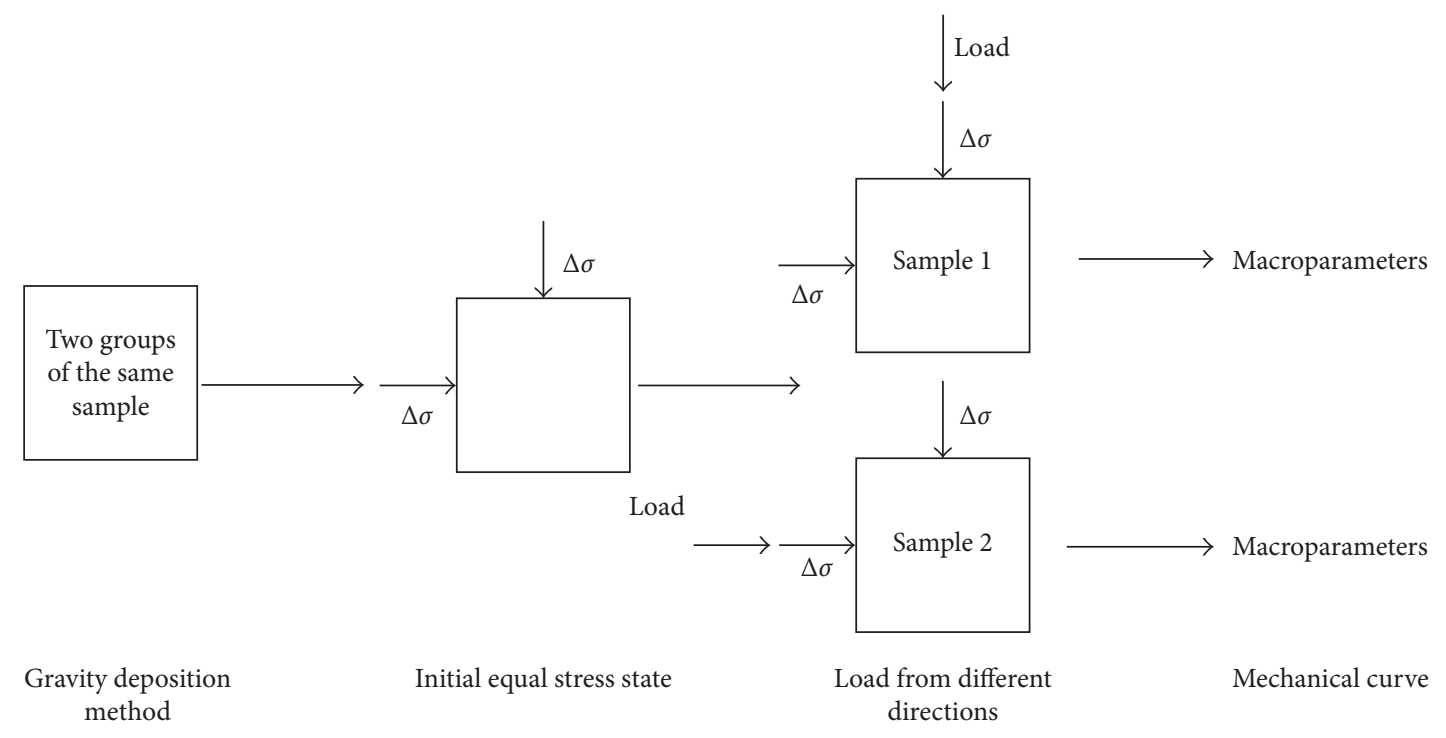

FIGURE 5: Stress paths of inherent anisotropy.

TABLe 6: Sample number and load condition.

\begin{tabular}{lcc}
\hline Sample number & Initial stress & Stress increment direction \\
\hline D1 & $\sigma_{10}=200 \mathrm{kPa}$ & Horizontal \\
D2 & $\sigma_{30}=150 \mathrm{kPa}$ & Vertical \\
E1 & $\sigma_{10}=250 \mathrm{kPa}$ & Horizontal \\
E2 & $\sigma_{30}=150 \mathrm{kPa}$ & Vertical \\
F1 & $\sigma_{10}=300 \mathrm{kPa}$ & Horizontal \\
F2 & $\sigma_{30}=150 \mathrm{kPa}$ & Vertical \\
\hline
\end{tabular}

particle and the horizontal direction, and the ordinate is the proportion of the total particles in a certain range of angles.

Figures $8(a)-8(c)$ show the distribution of the major axis orientation of the particles after different deposition processes. With the development of gravity deposition, the major axis orientation of the irregular unit changes obviously. In the angle range of $(-90,-60)$ and $(60,90)$, particle major axis orientation was significantly reduced, and there was a decrease in the range of $(-60,-30)$, but in the angle range of $(30,60)$ and $(-30,0)$, the particle major axis orientation was significantly increased. It is shown that the microstructure of the specimen changes under the action of gravity deposition, so that the arrangement of the particles in the sample is different in the horizontal direction and the vertical direction because the long-axis orientation distribution of the irregularly shaped granular unit is closer to the horizontal direction and make the contact of the particles in the direction of gravitational deposition tighter. This leads to the stress peak of the specimen in the vertical deposition direction, and the initial elastic modulus is larger than the horizontal direction, which is consistent with the principle of the inherent anisotropy.

4.1.2. The Effect of the Average Coordination Number. Figures 9(a)-9(c) show the curves of the average coordination number in the sample during the loading process when the strain is less than $1 \%$.
The average coordination number in PFC refers to the average number of particles in contact with the surroundings in a measurement circle. It is used to describe the stability of particle samples which states that the greater the coordination number, the closer the contact between particles, and the more difficult the compression of the particle becomes. Thus, the sample is kept more stable in this direction. It can be seen from Figure 9 that when the load is applied from the horizontal and vertical directions, the change of the average coordination number of the particles is not the same. When the load is applied from the vertical direction (gravity deposition direction), the average coordination number of the particles at the same strain is higher than the average of it obtained from the horizontal direction. The greater the number of particles in contact with each other in a measurement circle, the more stable the sample becomes, so when it is loaded from the vertical direction, the sample stability in the vertical direction is higher, and therefore, the sample has a smaller compression capacity in the vertical direction. At the same time, the initial elastic modulus and the stress peak in the vertical direction of Figures $7(\mathrm{a})-7(\mathrm{c})$ are larger than those in the horizontal direction.

Figures 9(a)-9(c) show that with the increase of the number of gravity deposition steps, the contact coordination number increases too, indicating that the greater the number of gravity deposition steps, the particle contact becomes closer, and therefore, the compression of the initial modulus and the stress peak will be greater too.

4.2. Analysis of Microcosmic Mechanism of the Stress-Induced Anisotropy. Stress-induced anisotropy is observed in specimens after being subjected to unequal initial stress states, and the anisotropy showed that the elastic modulus and stress peak in the direction of minimum principal stress were larger than those of the maximum principal stress. This is significantly different from the inherent anisotropy. 


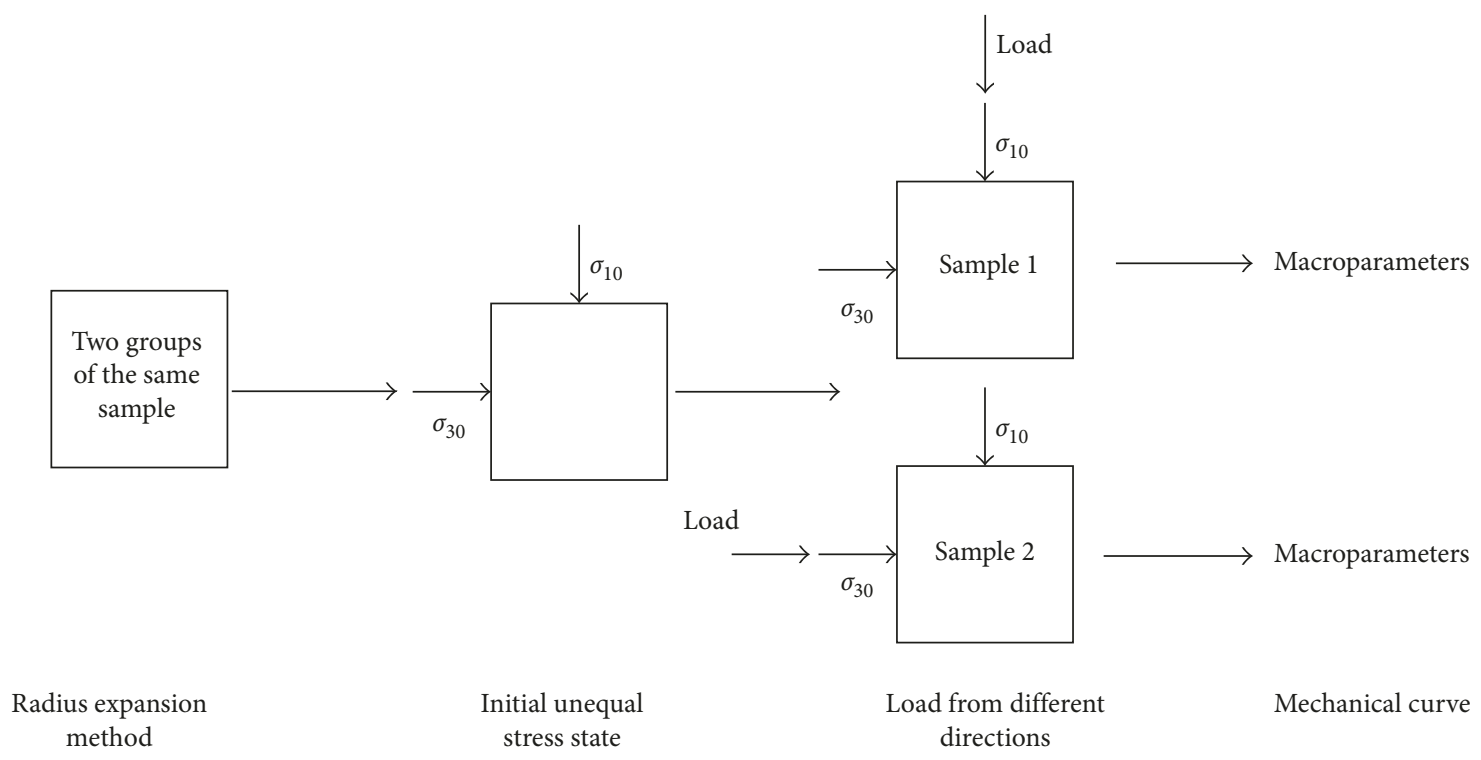

Figure 6: Stress path of induced anisotropy.

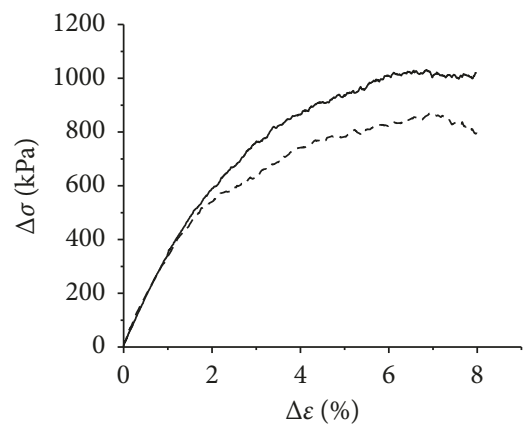

_ Vertical loading

- - - Horizontal loading

(a)

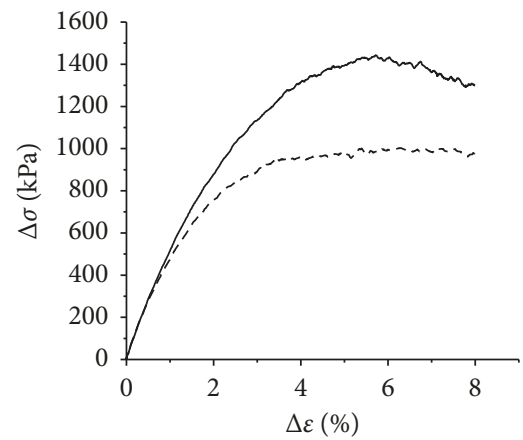

_ Vertical loading

- - - Horizontal loading

(c)

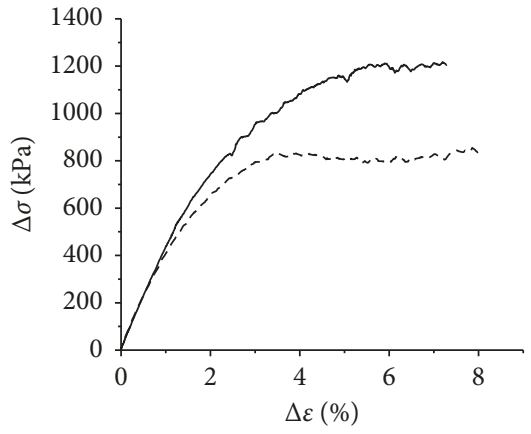

_ Vertical loading

- - - Horizontal loading

(b)

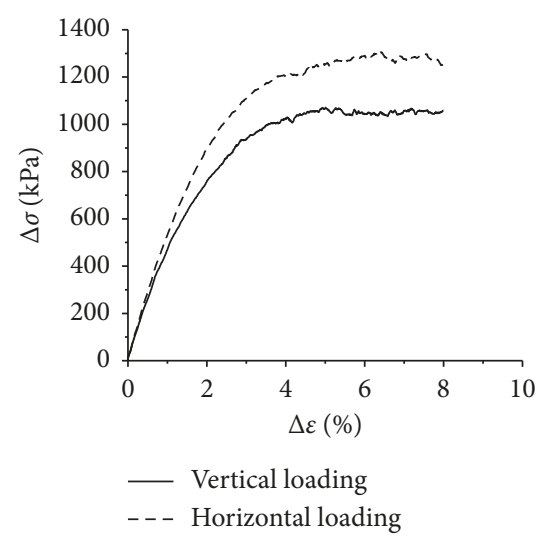

(d)

Figure 7: Continued. 


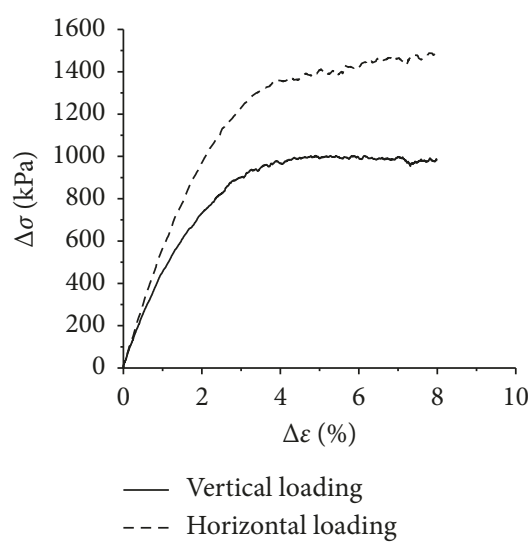

(e)

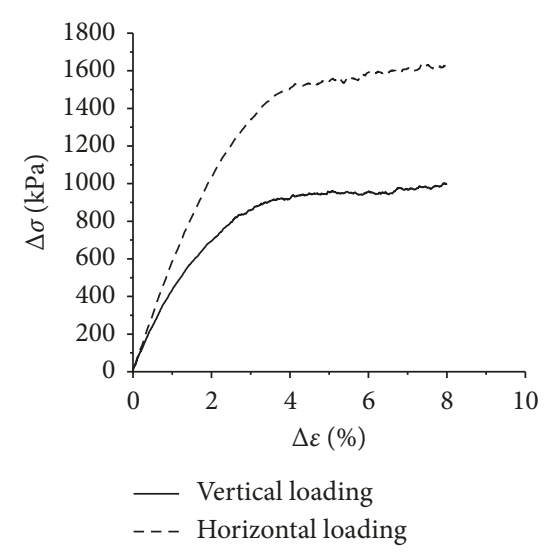

(f)

Figure 7: Curves of stress-strain relationship of three samples: (a) samples A1 and A2; (b) samples B1 and B2; (c) samples C1 and C2; (d) samples D1 and D2; (e) samples E1 and E2; (f) samples F1 and F2.

TABLE 7: Macromechanical parameters of samples.

\begin{tabular}{lcc}
\hline Sample number & Initial modulus $(\mathrm{MPa})$ & Stress peak $(\mathrm{kPa})$ \\
\hline A1 & 33.8 & 873 \\
A2 & 34.8 & 1030 \\
B1 & 40.7 & 856 \\
B2 & 43.8 & 1250 \\
C1 & 47.5 & 1001 \\
C2 & 51.7 & 1443 \\
D1 & 50.3 & 1303 \\
D2 & 43.9 & 1070 \\
E1 & 53.9 & 1490 \\
E2 & 41.8 & 1003 \\
F1 & 56.1 & 1636 \\
F2 & 39.6 & 1002 \\
\hline
\end{tabular}

4.2.1. The Effect of Major Axis Orientation on the Particles. In the study of above inherent anisotropy, the particles were arranged along the particle center line axis before deposition occurred under the action of gravity. In the stress-induced anisotropy test, there is no gravity deposition step. The following is an example of the F1 and F2 samples to analyze the variation of the center line axis orientation of the particles in the stress-induced anisotropy test, as shown in Figure 10.

Figure 10 shows the change of the major axis orientation of the particles with the load increment in the horizontal direction (minimum principal stress direction) or the vertical direction (maximum principal stress direction), and the particles do not produce obvious layer arrangement. This indicates that, for the stress-induced anisotropy of the specimen, the long-axis orientation of the irregular element particles is not the main cause of the sample's anisotropy in the horizontal and vertical directions. For stress-induced anisotropy, before the application of unequal stress, the sample had formed a certain degree of density, the particle unit contact was close, and the friction was relatively larger. The force is applied through the plate or test instrument at the end rather than applying directly to each particle above, so the particles will have a greater rotation and larger frictional resistance. For the inherent anisotropy, the major axis orientation of the particle unit changes obviously after the sample is completely formed. However, for the stressinduced anisotropy, due to the large frictional and rotational torque, the energy of the particle itself is small and the center line axis orientation of the particle unit is difficult to change, so the change of the center line axis orientation of the particle contributes little to the stress-induced anisotropy.

4.2.2. The Effect of the Average Coordination Number. In Figure 11, the curves of the average coordination number are presented. The specimens are in the same initial stress state, since the initial stress is not equal in the horizontal and vertical directions, and the average coordination number of particles varies with the strain increment when they are loaded from the horizontal and vertical directions. When the load is applied from the horizontal direction (minimum principal stress direction), the average coordination number of the same strain particles is higher than the average number of particles in the vertical direction (maximum principal stress direction). The larger average coordination number stabilizes the sample more; so when it is loaded from the minimum principal stress direction due to the maximum principal stress constraints, the sample stability is higher. Hence, there will be an initial modulus and stress peak of the minimum principal stress direction which is higher than those of the maximum principal stress direction. In addition, as shown in Figures 11(a)-11(c), the difference between the initial stress in the horizontal and vertical directions is large. The greater the coordination number obtained in the horizontal direction is, the smaller the coordination number obtained in the vertical direction becomes. It is more difficult to compress the sample from the horizontal direction, but from the vertical direction, the opposite is observed. Therefore, the sample will show macroscopically that, with the increase of the initial anisotropy stress, the difference between the initial elastic modulus and the stress peak in the horizontal direction and the vertical direction is larger, as shown in Figures 7(d)-7(f). 


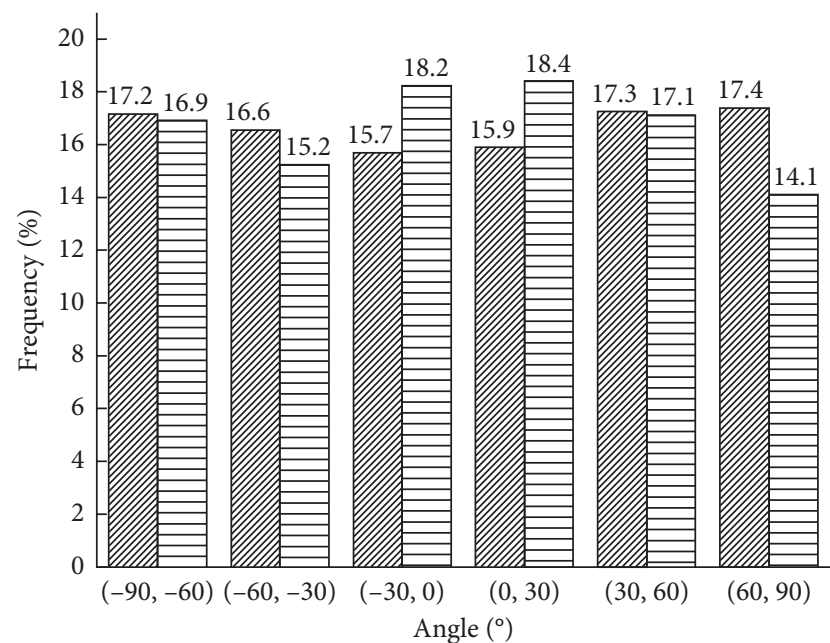

Before gravitational deposition After gravitational deposition

(a)

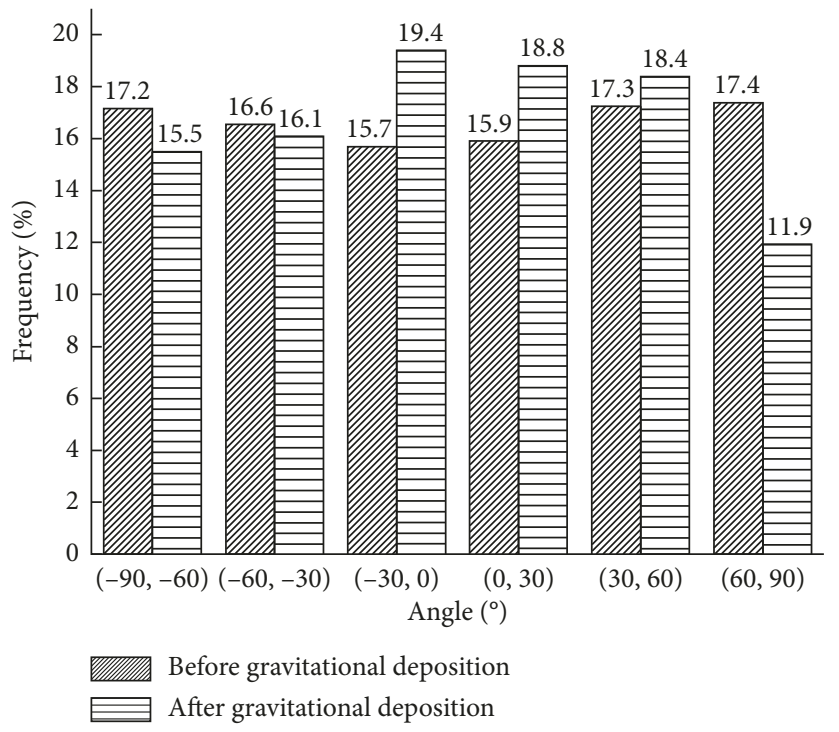

(b)

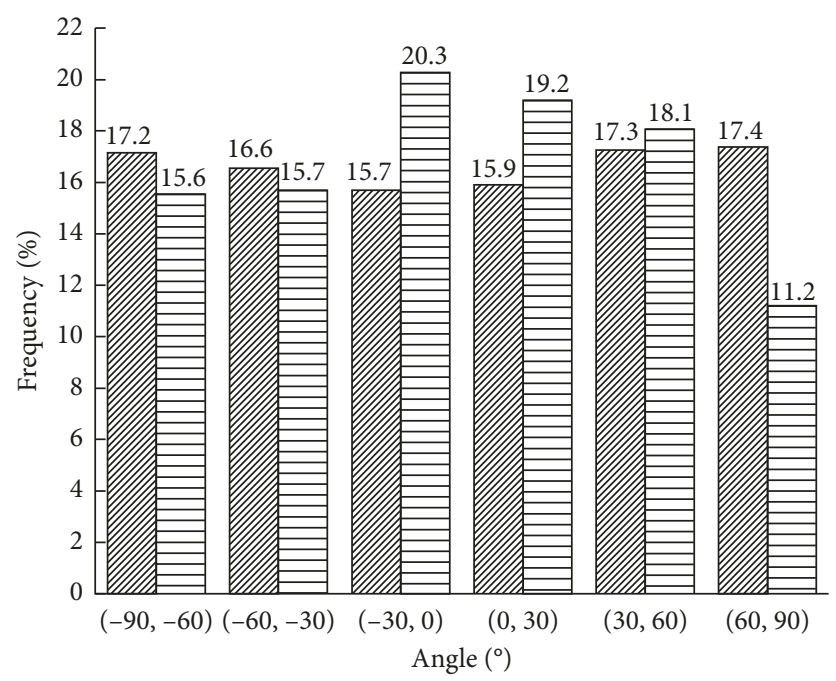

Before gravitational deposition

Е After gravitational deposition

(c)

FIGURE 8: Distribution of the major axis orientation of particles: (a) 9,000 deposition steps; (b) 12,000 deposition steps; (c) 15,000 deposition steps.

4.2.3. The Effect of the Contact Force between the Particles. The microscopic mechanism of stress-induced anisotropy in soil is also reflected in the contact force distribution. During the loading process, the contact force variation between the particles in the sample is different when the samples are loaded from the horizontal direction and the vertical direction. Figures 12 and 13 show the distribution of particle normal contact force and direction during the process of loading from horizontal and vertical directions when the samples F1 and F2 are in the initial stress state of $300 \mathrm{kPa}$ and $150 \mathrm{kPa}$. Each dot in the graph represents contact force between two particles, and the distance from the origin of the coordinates represents the magnitude of the contact force. The different quadrants and dots of different positions also represent the direction of the contact force. The more the dots are in the vertical direction, the more inclined this distribution is. In addition, the figure also gives the horizontal and vertical contact force of the average. When the strain is 0 (before loading), the horizontal and vertical directions of the normal distribution are oval, and the major axis is in the vertical direction. This shows that the distribution of the particle normal contact force and direction are the same before the loading of the two groups, as shown in Figures 12(a) and 13(a). But when the two groups of samples were loaded from the horizontal and vertical directions, the distribution of the particle normal contact force and direction was significantly different with the strain increment. When the load is applied in the horizontal direction 


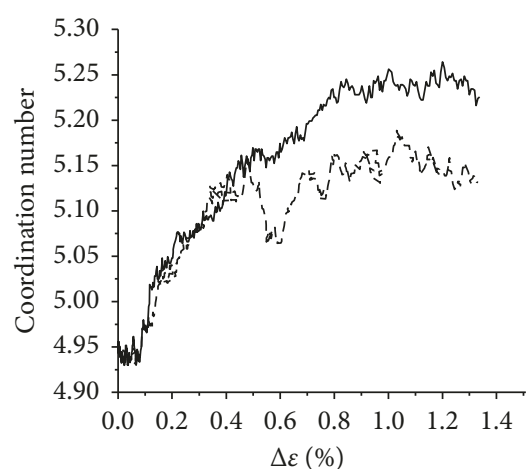

- Vertical loading

- - - Horizontal loading

(a)

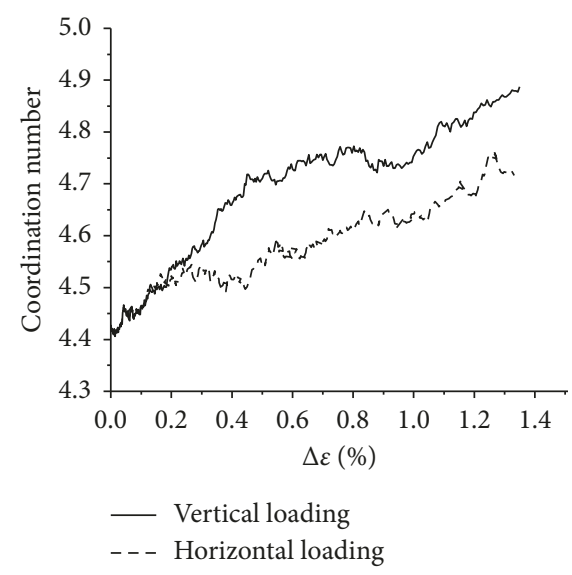

(b)

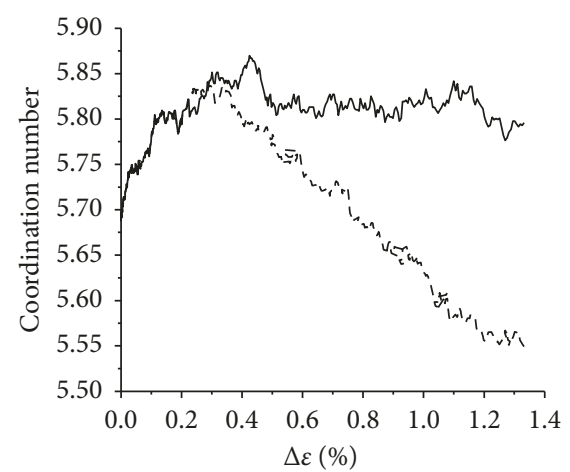

- Vertical loading

- - - Horizontal loading

(c)

Figure 9: Curves of coordination numbers: (a) sample group A; (b) sample group B; (c) sample group C.

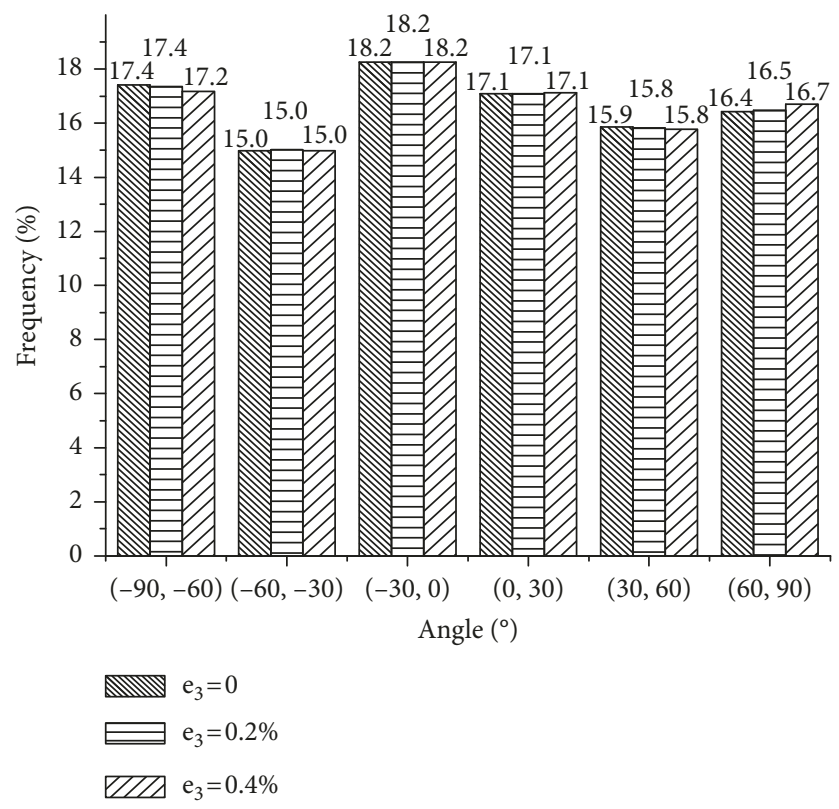

(a)

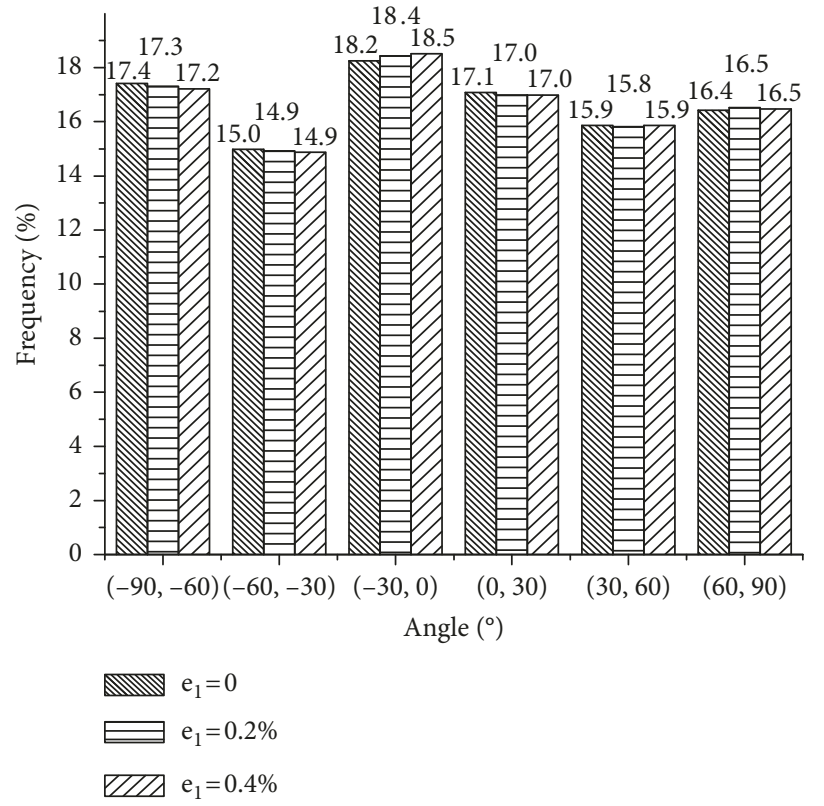

(b)

FIgURE 10: Distribution of major axis orientation of particles: (a) F1 sample long axis orientation change; (b) F2 sample long axis orientation change.

(minimum principal stress direction), the normal contact force in the horizontal direction increases rapidly with the increase of the strain, and the vertical direction (the direction of the maximum principal stress) changes slightly. The distribution of the contact force gradually changes from elliptical to circular. This means that the advantage of the distribution of the contact force in the vertical direction tends to reduce as the loading progresses. As the strain increment continues to increase, the contact force in the horizontal direction starts to exceed the contact force in the vertical direction. The particle movement is difficult in a relatively stable state, so the elastic modulus and peak stress are larger when loading from the horizontal direction. When the load is applied in the vertical direction, the distribution of particle normal contact force gradually increases, the difference in the contact force between the vertical and horizontal directions of the particles increases from the beginning, and the movement of the particles is easy. When it is loaded from the vertical direction, the specimen's modulus and stress peaks will be lower than the relevant parameters obtained from the horizontal direction.

4.3. The Differences and Relations between the Two Kinds of Anisotropy. Analyses are conducted on the two kinds of anisotropy of soil in detail, and there are some relations and differences between them. The microscopic mechanism of 


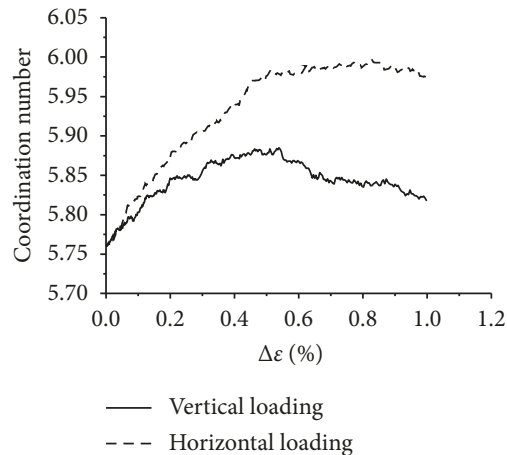

(a)

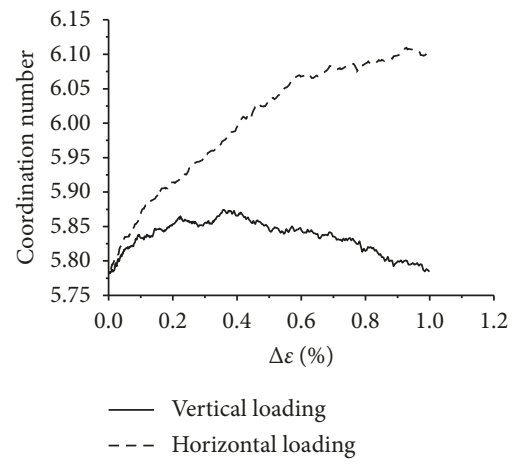

(b)

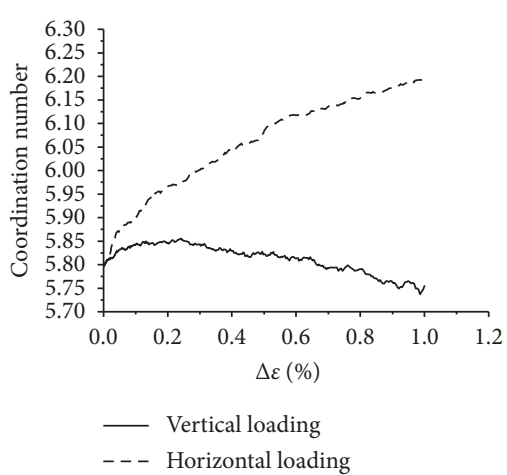

(c)

FIgURE 11: Curves of the average coordination number: (a) sample group A; (b) sample group B; (c) sample group C.

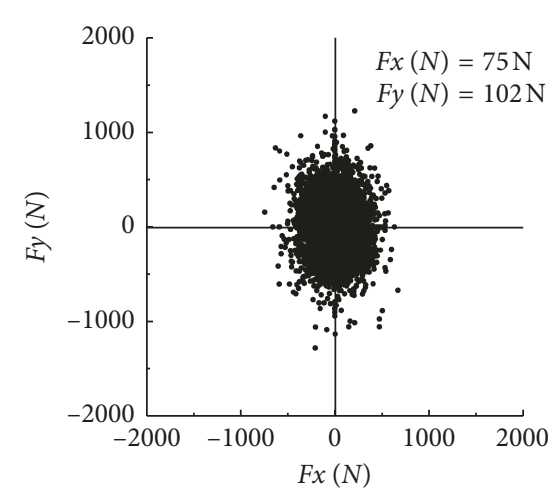

(a)

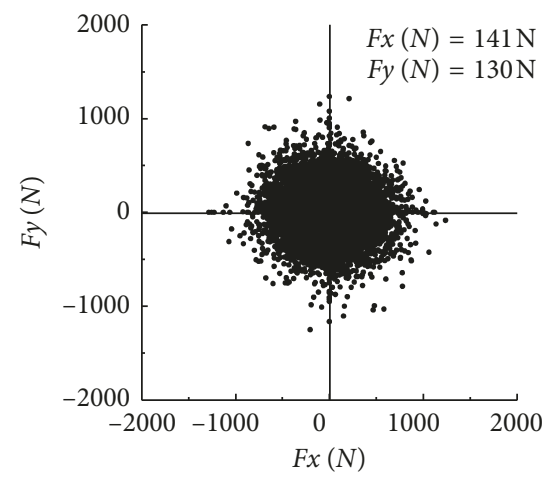

(b)

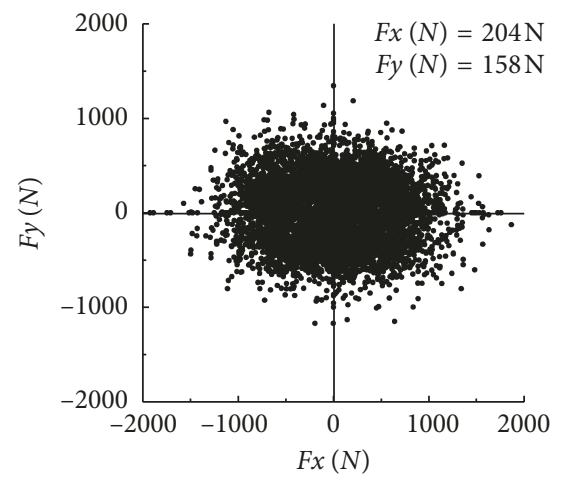

(c)

FiguRE 12: Distribution of the particle normal contact force and direction during the process of sample F1 loading: (a) $\varepsilon_{3}=0$; (b) $\varepsilon_{3}=0.4 \%$; (c) $\varepsilon_{3}=0.8 \%$.

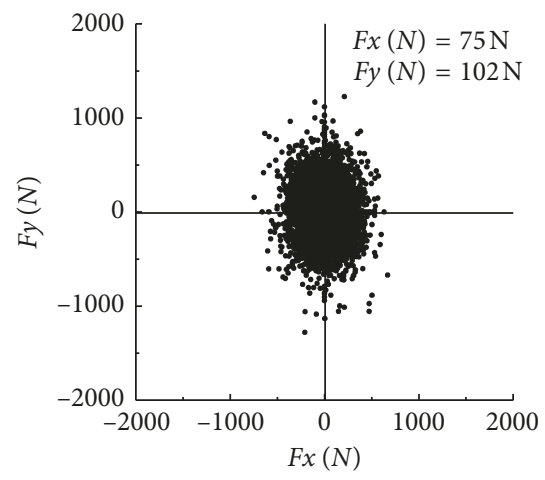

(a)

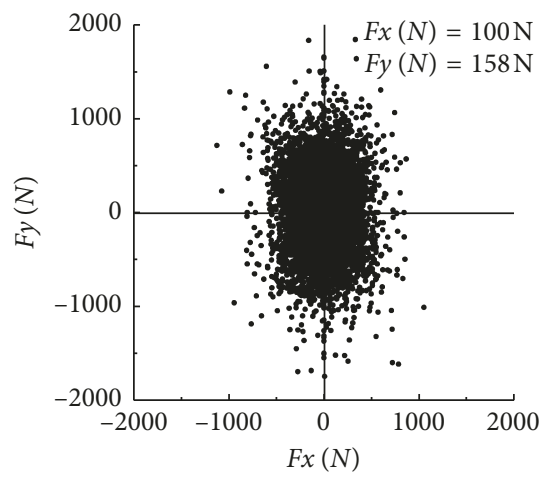

(b)

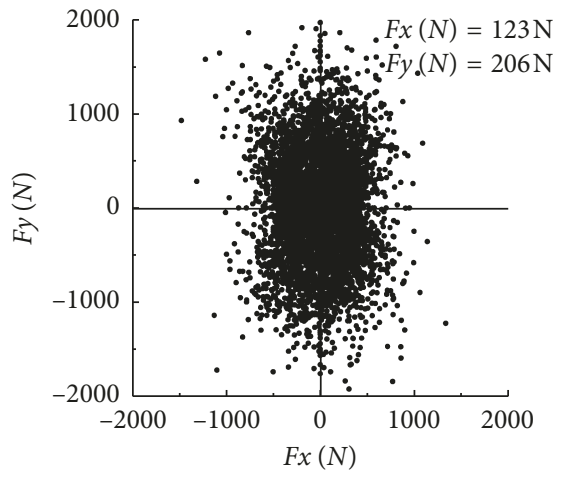

(c)

FIGURE 13: Distribution of the particle normal contact force and direction during the process of sample F2 loading: (a) $\varepsilon_{1}=0$; (b) $\varepsilon_{1}=0.4 \%$; (c) $\varepsilon_{1}=0.8 \%$.

the two kinds of anisotropy is uniform. Both the inherent anisotropy and the stress-induced anisotropy are mainly caused by the different distribution and arrangement in different directions in soil microstructure, such as directional distribution of major axis of the particle unit, the arrangement of particles in space pore distribution, and the average coordination number of particles.
However, there are some differences between the forms of expression of the anisotropy. In the course of deposition, the particles easily rotate and loose contact between each other. When the particles are subjected to gravity, the layers are arranged in the horizontal direction, resulting in tight contact in the vertical direction and difficulty in compression. The stress peak and the elastic modulus in the vertical 
direction are larger than those in the horizontal direction, which are called inherent anisotropy. The contact between particles is not lost as the before gravity deposition condition, and there is a strong force between particles and hence it is not easy to rotate; this is called the stress-induced anisotropy. The soil sample is subjected to an unequal initial stress state, resulting in differences in the horizontal and vertical distributions of the contact force and the coordination number. In the late loading process in the vertical direction (maximum principal stress direction), the shear trend increases from the commencement of loading. In the horizontal direction (minimum principal stress direction), the shear trend of sample decreases first and thereafter increases. The sample undergoes a gradual compression and stability process. Therefore, the stress peak and elastic modulus in the final horizontal direction are larger than those in the vertical direction.

\section{Conclusion and Discussion}

As a discontinuous discrete material, the mechanical properties and research methods of sandy materials are quite different from those of continuous materials. Because of the discreteness of the particle structure the sandy materials show an obvious anisotropy on the macroscopic scale under the action of gravity or under the condition of unequal stress. The generation of this anisotropy is also closely related to the microstructural properties of the bulk material. In view of the limitations of the research methods of discontinuous discrete materials and the complexity of laboratory tests, the numerical simulation software of particle flow (PFC2D) was adopted in this paper. A group of plane biaxial specimens was established by gravity deposition and radius expansion method. Stress and strain curves and macromechanical parameters in different directions under different conditions were obtained by loading in horizontal and vertical directions. The macroscopic anisotropy was analyzed and explained from the microscopic point of view, using the parameters of particle orientation, and coordination number, and the magnitude of the particle indirect contact force as an index. The conclusions are as follows:

(1) In this paper, numerical results of an irregular element are closer to the results of the triaxial test of the standard sand sample. The proposed irregular unit is more suitable for the shape characteristics of soil particles than the original disk unit in PFC. Based on this numerical specimen, a numerical test of a more extensive stress path can be carried out.

(2) In the process of gravity deposition, the particle units are obviously arranged in layers. This arrangement gives the soil more contact and compression in one direction than in the other. In the later loading process, the soil shows obvious anisotropic mechanical characteristics.

(3) The stress-induced anisotropy of soil is produced by the interaction of the irregular internal structure and external unequal stress. The change of the internal structure of the soil is concomitant with the change of the external stress state. The fundamental reason for the anisotropy is that the microstructure of the material has undergone uneven changes, but the reason for this change is the role of external load.

(4) The reasons for the two kinds of anisotropy can be attributed to the inhomogeneous changes of $\mathrm{mi}-$ crostructure and to the micromechanical mechanism, but there are differences in their forms of expression.

(5) In this paper, the PFC simulation of sand inherent and stress-induced anisotropy is carried out. It is suggested that a scale numerical test tool will be established by numerical simulation of the completed macrounit tests. The reliability of the discrete element numerical tools is preliminarily verified by comparing with the results of unit tests. The numerical experiments under more extensive stress path can be carried out, and the stress and strain of granular materials under complex stress conditions are obtained. It provides a reference for the macroscopic mechanism of the macroscopic mechanical behavior of granular materials and for establishing the macroscopic constitutive model of rock.

\section{Data Availability}

The data that support the findings of this study are available from the corresponding author upon reasonable request.

\section{Conflicts of Interest}

The authors declare that they have no conflicts of interest.

\section{Acknowledgments}

The work was supported by the project of National Natural Science Foundation of China (nos. 41530637 and 51578214).

\section{References}

[1] A. V. Abelev and P. V. Lade, "Effects of cross anisotropy on three-dimensional behavior of sand I: stress-strain behavior and shear banding," Journal of Engineering Mechanics, vol. 29, no. 2, pp. 160-166, 2003.

[2] J. R. F. Arthur and B. K. Menzies, "Inherent anisotropy in a sand," Geotechnique, vol. 22, no. 1, pp. 115-128, 1972.

[3] K. Y. Zhang, Study and Application on Soil's Constitutive Model with the Consideration of Stress-Induced Anisotropy, Hohai University, Nanjing, China, 2004, in Chinese.

[4] J. R. F. Arthur, K. S. Chua, and T. Dunstan, "Induced anisotropy in a sand," Geotechnique, vol. 27, no. 1, pp. 13-30, 1977.

[5] K. Y. Zhang and F. N. Charkley, "An anisotropic constitutive model of geomaterials based on true triaxial testing and its application," Journal of Central South University, vol. 24, no. 6, pp. 1430-1442, 2017.

[6] K. Y. Zhang and Z. Z. Yin, "Discussion on soil's anisotropy under complicated stress state and the study method," Rock and Soil Mechanics, vol. 28, pp. 149-154, 2007. 
[7] E. Hoque and F. Tatsuoka, "Anisotropy in elastic deformation of granular materials," Soils and Foundations, vol. 38, no. 1, pp. 163-179, 1998.

[8] Itasca Consulting Group, Inc., PFC2D Particle Flow Code in 2 Dimensions Version 5.0, Itasca Consulting Group, Inc., Minneapolis, MN, USA, 2014.

[9] P. A. Cundall, "A computer model for simulating progressive large-scale movement in blocky rock systems," in Proceedings of Symposium of the International Society of Rock Mechanics, vol. 1, Nancy, France, 1971.

[10] A. T. Procopio and A. Zavaliangos, "Simulation of multi-axial compaction of granular media from loose to high relative densities," Journal of the Mechanics and Physics of Solids, vol. 53, no. 7, pp. 1523-1551, 2005.

[11] P. A. Cundall, "A discontinuous future for numerical modelling in geomechanics," in Proceedings of the Institution of Civil Engineers-Geotechnical Engineering, vol. 149, London, 2001.

[12] Y. Chi, A Study of the Mechanical Properties of the Soil-A Simulation of the Particle Flow in the Shear Zone of the StressStrain Relationship, Tongji University, Shanghai, China, 2002.

[13] M. J. Jiang, D. Peng, Z. F. Shen et al., "DEM analysis on formation of shear band of methane hydrate bearing soils," Journal of Geotechnical and Geoenvironmental Engineering, vol. 36, no. 9, pp. 1624-1630, 2014.

[14] C. X. Tong, M. Zhou, L. W. Zhang et al., "Numerical modelling of biaxial compression tests for granular materials with inherent anisotropy using DEM," Chinese Journal of Rock Mechanics and Engineering, vol. 33, no. 2, pp. 4227-4232, 2014, in Chinese.

[15] D. D. Shi, Micromechanical Simulations of Sand Behavior under Monotonic and Cyclic Loading, Tongji University, Shanghai, China, 2007. 


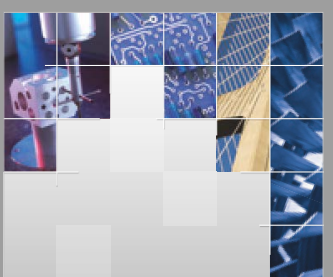

\section{Enfincering}
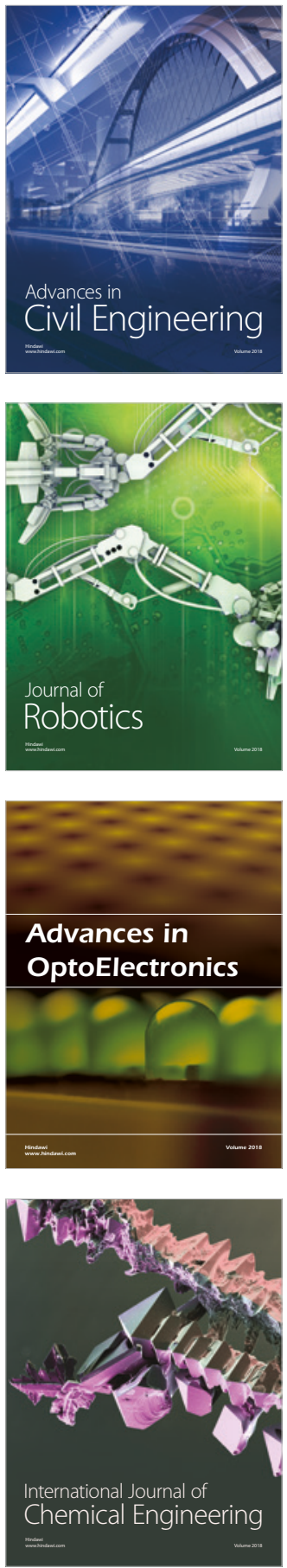

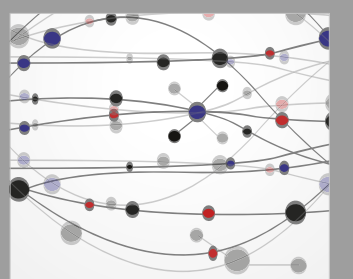

\section{Rotating \\ Machinery}

The Scientific World Journal

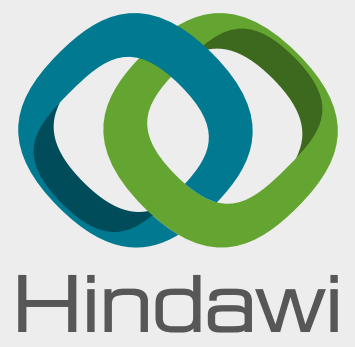

Submit your manuscripts at

www.hindawi.com
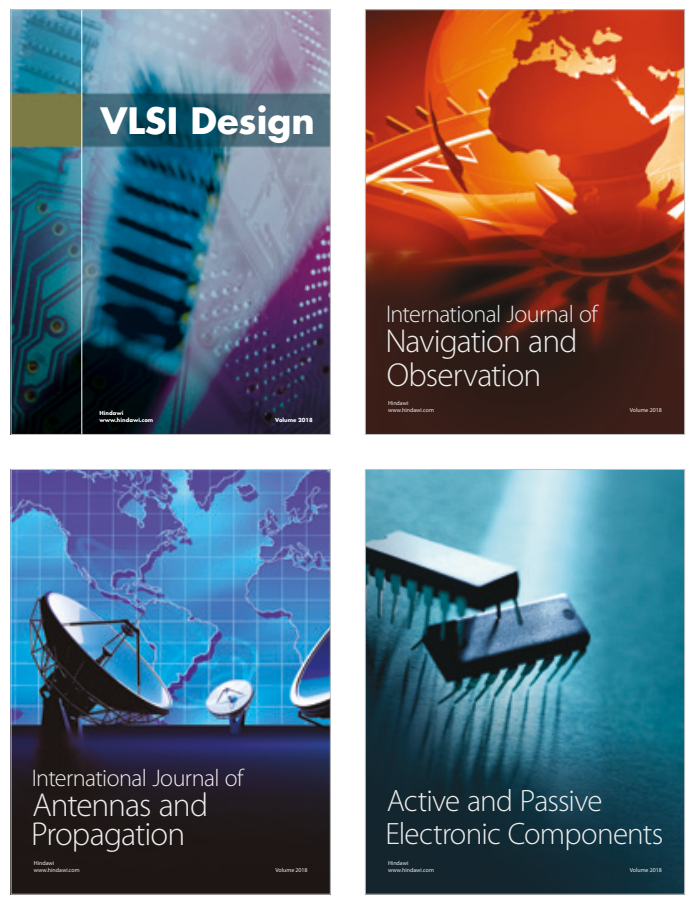
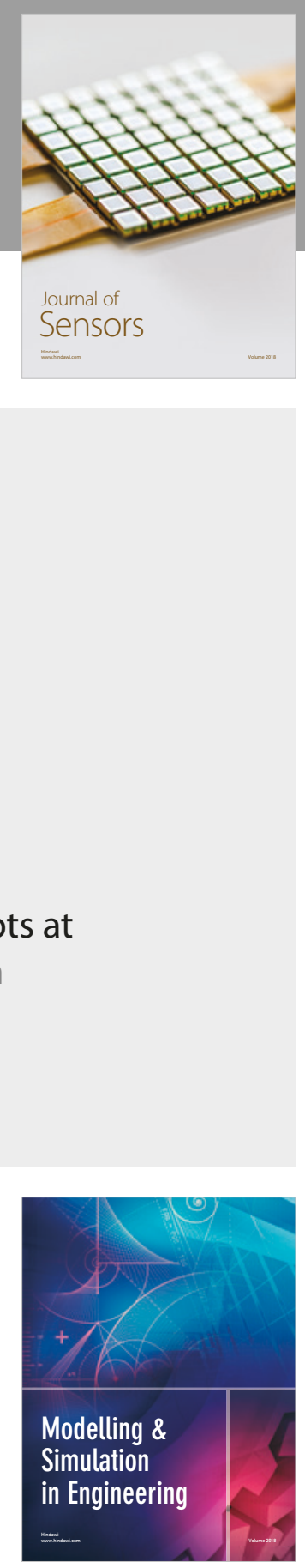

\section{Advances \\ Multimedia}
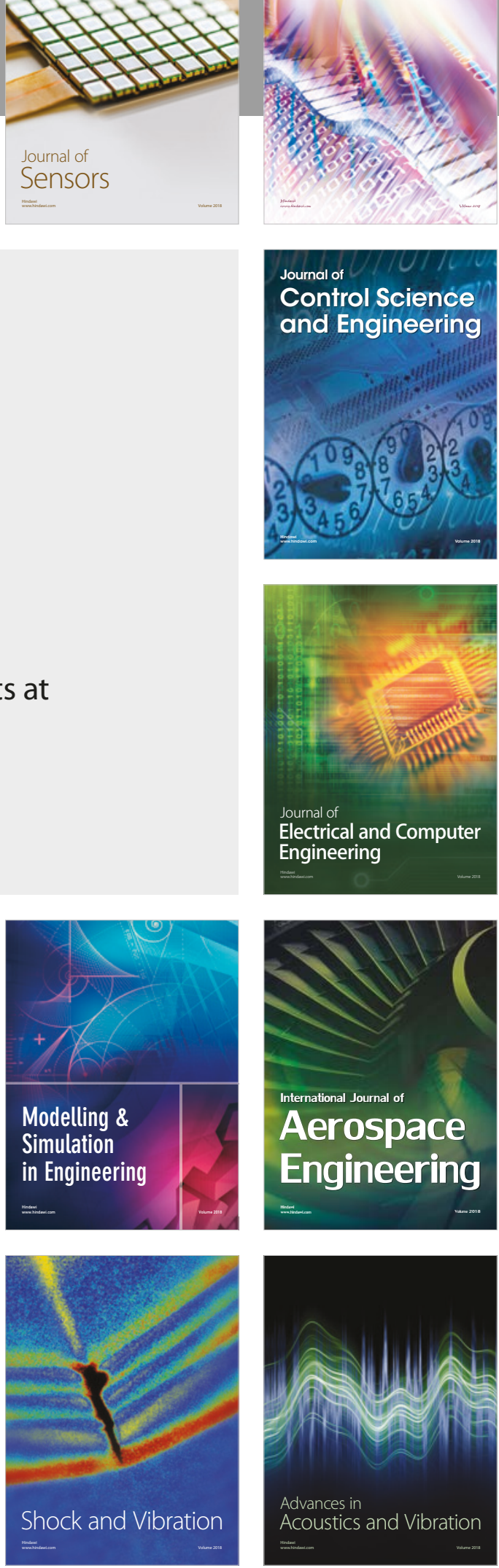